\title{
Statistical inference for evolving periodic functions
}

\author{
Marc G. Genton \\ University of Geneva, Switzerland, Texas A\&M University, College Station, USA, and \\ Australian National University, Canberra, Australia \\ and Peter Hall \\ Australian National University, Canberra, Australia
}

[Received September 2005. Final revision February 2007]

\begin{abstract}
Summary. In the study of variable stars, where the light reaching an observer fluctuates over time, it can be difficult to explain the nature of the variation unless it follows a regular pattern. In this respect, so-called periodic variable stars are particularly amenable to analysis. There, radiation varies in a perfectly periodic fashion, and period length is a major focus of interest. We develop methods for conducting inference about features that might account for departures from strict periodicity. These include variation, over time, of the period or amplitude of radiation. We suggest methods for estimating the parameters of this evolution, and for testing the hypothesis that the evolution is present. This problem has some unusual features, including subtle issues of identifiability.
\end{abstract}

Keywords: Amplitude; Astronomy; Bandwidth; Bootstrap; Kernel methods; Light curve; Non-parametric regression; Parametric inference; Time transformation

\section{Introduction}

The light that is emitted by some stars in the heavens varies very nearly periodically with time. However, in other cases there is evidence that the 'light curve', as it is sometimes called, is not quite periodic. Either the period or the amplitude of radiation evolves slowly over time, even if the basic underlying shape of the curve does not alter. For discussion of these issues see, for example, Eyer and Genton (1999), Koen (2005), Rodler and Guggenberger (2005), Sterken (2005) and Hart et al. (2007).

Physical considerations suggest that the nature of period or amplitude evolution might be relatively simple. In particular, either quantity may slowly increase or decrease over time, perhaps linearly. Departures from linear change might be explainable in terms of low degree polynomials, e.g. quadratics.

Of course, in the context of changes to period, an astronomer envisages adjustments that occur in the continuum, rather than discretely. Therefore, a function that is 'basically' periodic, but has a changing period, is modelled by applying a periodic function to another function that represents a monotone, continuous time change.

Problems of this nature have unusual subtle aspects, which should guide our approaches to modelling and inference. In particular, any positive function can be derived from any other by simply changing its amplitude over time. Substantial changes in the shape of a periodic function can be produced by merely altering the period, without changing the amplitude.

Address for correspondence: Marc G. Genton, Department of Statistics, Texas A\&M University, College Station, TX 77843-3143, USA.

E-mail: genton@stat.tamu.edu

(C) 2007 Royal Statistical Society

$1369-7412 / 07 / 69643$ 
These issues might be thought to argue in favour of a parametric, rather than non-parametric, treatment, carefully constructed to avoid the problems of identifiability that were foreshadowed above. However, the very rich variety of light curves that are observed in even periodic settings suggests that a highly adaptive non-parametric argument should lie at the heart of any broadly applicable approach to inference about evolving periodic functions.

We shall take this view, modelling the periodic function in a non-parametric way but assuming simple parametric models for period and amplitude change. This approach allows us to estimate the periodic function with non-parametric convergence rates, although we may estimate the period at rate $n^{-3 / 2}$, where $n$ denotes sample size. This high degree of accuracy reflects the fact that very small mathematical departures from the correct period can lead to large visual departures in the data.

Our work is motivated by data on the Mira variable R Hydrae, a long period variable star of which the period and amplitude have been evolving through time. Fig. 1 depicts data on the magnitude (brightness) of this star during 1900-1950 (Fig. 1(a)) and 1950-2001 (Fig. 1(b)) with an irregular time design. On the basis of these data, and using wavelet methods, Zijlstra et al. (2002) reported a decline in the period of R Hydrae, from about 420 to 380 days during 1900-1950, accompanied by a decline in its semiamplitude from magnitude 2.4 to 1.7. Zijlstra et al. (2002) also concluded that the period and semiamplitude of R Hydrae stabilized during 1950-2001, to about 385 days and magnitude 1.7 respectively. From Fig. 1, it is difficult to validate these claims visually. We shall investigate them by using the methods that are developed in this paper.

There is a large literature on statistical analysis of data on variable stars. The method that is most favoured among astronomers is based on the periodogram and can be traced to influential work of Barning (1963), Lomb (1976), Ferraz-Mello (1981) and Scargle (1982). Recent instances of its application are to be found in the contributions of de Cat and Aerts (2002), DePoy et al.

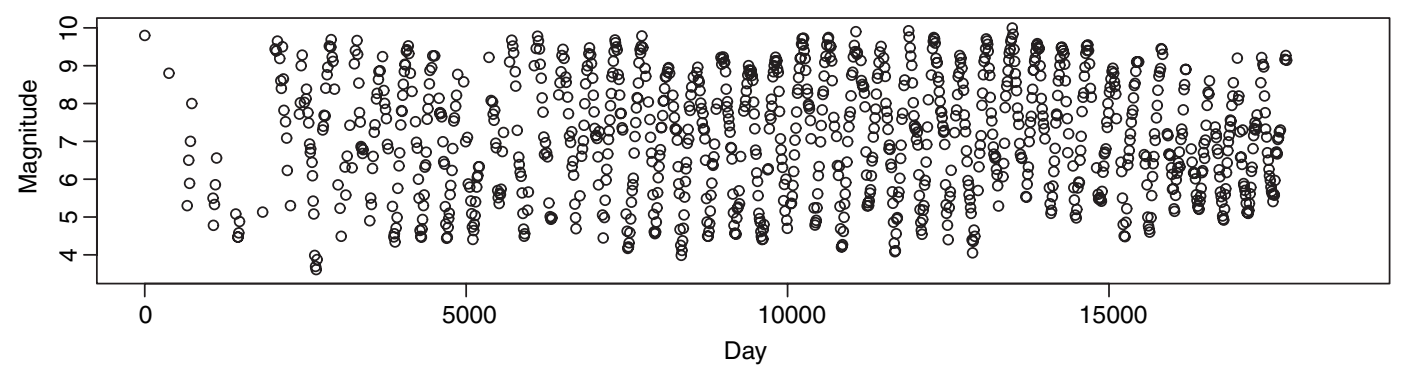

(a)

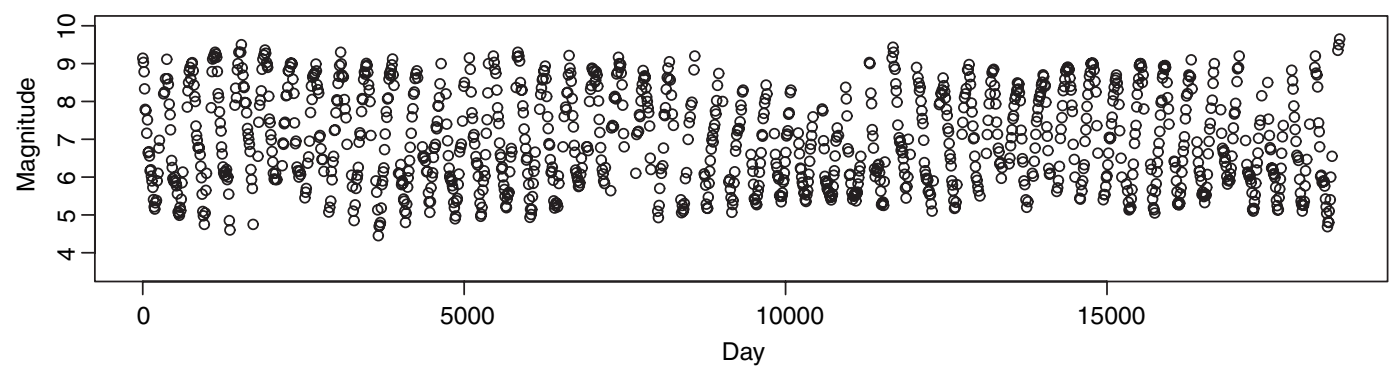

(b)

Fig. 1. Light curve of the Mira variable R Hydrae: (a) 1900-1950; (b) 1950-2001 
(2004), Lanza et al. (2004), Aerts and Kolenberg (2005), Maffei et al. (2005) and Hall and Li (2006).

Evolving periods might also be tackled by using the notion of time-varying spectra; see, for example, Dahlhaus (1997) and Neumann and von Sachs (1997). However, we shall take a time domain approach, as in the work of Hall et al. (2000) and Hall and Yin (2003).

This paper is organized as follows. In Section 2, we describe various models for functions with evolving period and amplitude, and discuss methodology for statistical inference in this setting. We illustrate the numerical properties of our methods in Section 3, using simulated and real data. In Section 4 we outline theoretical properties, including convergence rates. All proofs are collected together in Appendix A.

\section{Models and methodology}

\subsection{Functions with evolving period and amplitude}

Let $g_{0}$ denote a periodic function with unit period, and let $t$ be a continuously differentiable, strictly increasing function. Represent time by $x$, and define $t_{x}=t(x)$ and $t_{x}^{\prime}=t^{\prime}(x)>0$. We shall consider $t$ to provide a change of time, from $x$ to $t_{x}$.

Against this background, assume that a function $g$ can be represented as

$$
g(x)=g_{0}\left(t_{x}\right) .
$$

We may think of $g$ as having period $1 / t_{x}^{\prime}$ at time $x$. Indeed, for small $u>0$,

$$
g(x+u)=g_{0}\left\{t_{x}+t_{x}^{\prime} u+o(u)\right\} .
$$

Since the function $d(u)=g_{0}\left(t_{x}+t_{x}^{\prime} u\right)$ has period $1 / t_{x}^{\prime}$, then, if the time transformation $t_{x+u}$ were to continue in a linear way for $u>0, g$ would have period $1 / t_{x}^{\prime}$ at all future times $x+u$. More generally, without the assumption of linearity, the function $g$ that is given by equation (1) can be considered to have a period $p_{x} \equiv 1 / t_{x}^{\prime}$ that evolves as time $x$ increases.

Amplitude, also, can evolve. Indeed, if $a>0$ is a smooth function, representing amplitude, and if we write $a_{x}$ for $a(x)$, then, from some points of view, we might generalize equation (1) to

$$
g(x)=a_{x} g_{0}\left(t_{x}\right) .
$$

In this case we might, perhaps, consider $g$ to have period $1 / t_{x}^{\prime}$, and amplitude $a_{x} g_{0}\left(t_{x}\right)$, at $x$.

The concept of evolving amplitude must be treated cautiously, however. Whereas changing time can alter only the distances between successive peaks and troughs in the function $g_{0}$, changing both amplitude and time can produce a function which is entirely different. In particular, any smooth, strictly positive function $g$ can be constructed as at equation (2), with $a>0$ denoting a smooth change in amplitude, $t_{x} \equiv x$ being the identity transformation, and $g_{0}$ denoting any strictly positive smooth function, periodic or otherwise.

A conclusion to be drawn from this discussion is that, unless amplitude is determined by a relatively simple parametric model, and unless it changes only very slowly over time, relative to the lengths of periods, it can interact too greatly with period to be interpretable independently of period.

It is possible for non-identifiability of $g_{0}$ to occur even when $a \equiv 1$ and the function $t$ has a simple parametric form. For example, suppose that, in the particular case $p=1$,

$$
t_{x+k p}=t_{x}+k \quad \text { for each } x \in[0,1] \text { and each integer } k \geqslant 1 .
$$

(We can think of expression (3) as defining a time change function $t$ with 'pseudoperiod' $p$.) Then, since $g_{0}$ has period 1, it follows that $g_{0}\left(t_{x+k}\right)=g_{0}\left(t_{x}\right)$ for each $x$ and each integer $k$. Therefore, the periodic function $g \equiv g_{0}(t)$ is representable as either a time-changed version of the 
function $g_{0}$ with unit period or more directly as the non-time-changed function $g_{1} \equiv g_{0}(t)$ with unit period. If we consider this particular time change function $t$, and also the identity time change $\iota(x) \equiv x$, to be members of a larger parametric class, $\mathcal{T}$ say, of time change functions, then there is ambiguity in determining the member $s$ of $\mathcal{T}$ that enables us to represent $g \equiv g_{0}(t)$ as $g=g_{2}(s)$ where $g_{2}$ has period 1 .

\subsection{Models for period}

We shall view equation (2) as a model for a regression mean function $g$, where the functions $a$ and $t$ are determined parametrically and $g_{0}$ is treated non-parametrically. For equation (2) to be readily interpretable in astronomical terms, it is helpful for the models for $t$ to be quite simple. For example, taking $t_{x}=\theta_{2}^{-1} \log \left(\theta_{1}+\theta_{2} x\right)+\theta_{3}$, for constants $\theta_{1}>0, \theta_{2}$ and $\theta_{3}$, implies that $1 / t_{x}^{\prime}=\theta_{1}+\theta_{2} x$. In this case the initial period is $\theta_{1}$, and the period increases linearly with time, with slope $\theta_{2}$. (Of course, $\theta_{2}<0$ implies decreasing period.) If we start measuring time at zero when $x=0$ then we require $\theta_{3}=-\theta_{2}^{-1} \log \left(\theta_{1}\right)$, and then the model becomes

$$
t_{x}=\theta_{2}^{-1} \log \left(1+\theta_{1}^{-1} \theta_{2} x\right) .
$$

We might refer to equation (4) as a 'linear model', since it results from a linear model for period. Analogously we could refer to the model

$$
t_{x}=\left(\theta_{1} \theta_{2}\right)^{-1}\left\{1-\exp \left(-\theta_{2} x\right)\right\}
$$

for which $1 / t_{x}^{\prime}=\theta_{1} \exp \left(\theta_{2} x\right)$, as an 'exponential model'. It is an attractive alternative to the linear model in certain cases. In particular, its period is unequivocally positive. The condition of positivity can be awkward to ensure in the case of a decreasing linear model for period. However, the exponential model is seldom physically reasonable if $\left|\theta_{2}\right|$ is large; only for small $\left|\theta_{2}\right|$ is it attractive, and there it is close to the linear model. On grounds of pragmatism, our numerical and theoretical work will be for the model

$$
t_{x}=\theta_{1}^{-1} x+\theta_{2} x^{2}+\ldots+\theta_{k} x^{k}, \quad 0 \leqslant x \leqslant n,
$$

where $k \geqslant 1$. An exponentiated version of equation (6) is also possible.

In models (4)-(6), and in a realistic theoretical setting where data are assumed to be observed at an approximately constant rate over a time interval $[0, n]$ of increasing length $n$, usually only the parameter $\theta_{1}$, representing period at time $x=0$, would be kept fixed as $n$ increased. The parameters $\theta_{2}, \ldots, \theta_{k}$ would typically decrease to 0 with $n$, and in fact would usually decrease at such a rate that $n^{j-1}\left|\theta_{j}\right|$ was at least bounded. This would prevent the period from changing by an order of magnitude over the observation-time interval.

\subsection{Models for amplitude}

Models for the function $a_{x}$ can be similar to those for $t_{x}$. However, to avoid identifiability problems we should insist that $a_{x}=1$ at the initial time, so that initial amplitude is incorporated in the function $g_{0}$ and not duplicated in $a_{x}$. Bearing this in mind, and taking the initial time to be $x=0$, potential models include

$$
a_{x}=1+\omega_{1} x+\ldots+\omega_{l} x^{l}, \quad 0 \leqslant x \leqslant n,
$$

and its exponentiated form, $a_{x}=\exp \left(\omega_{1} x+\ldots+\omega_{l} x^{l}\right)$. For both these models, theoretical analysis requires an assumption, such as $\sup _{n} \sup _{1 \leqslant j \leqslant l}\left(n^{j}\left|\omega_{j}\right|\right)<\infty$, to ensure that the amplitude does not alter by an order of magnitude over the observation time interval. 


\subsection{Model for data generation}

Assume that data $\left(X_{1}, Y_{1}\right), \ldots,\left(X_{n}, Y_{n}\right)$ are generated by the model

$$
Y_{i}=a\left(X_{i} \mid \omega^{0}\right) g_{0}\left\{t\left(X_{i} \mid \theta^{0}\right)\right\}+\varepsilon_{i},
$$

where $a_{x}=a(x \mid \omega)$ and $t_{x}=t(x \mid \theta)$ are smooth positive functions that are determined by finite vectors $\omega$ and $\theta$ of unknown parameters, $\omega^{0}$ and $\theta^{0}$ denote the true values of the respective parameters, $t(\cdot \mid \theta)$ is strictly increasing, $a(\cdot \mid \omega)$ is bounded away from 0 and $\infty$, and the experimental errors $\varepsilon_{i}$ are independent with zero means and uniformly bounded variances. In particular, $t(\cdot \mid \theta)$ and $a(\cdot \mid \omega)$ could be any one of the models that were introduced in Sections 2.2 and 2.3 respectively. In model (8), $Y_{i}$ denotes star brightness and is measured on a logarithmic scale in units of apparent magnitude.

As in Section 2.1, $g_{0}$ is assumed to be a smooth periodic function with period 1 . Therefore, even if the regression mean, $g(x)=a(x \mid \omega) g_{0}\{t(x \mid \theta)\}$, were a conventional periodic function, without any amplitude or time change, the period, $p$ say, would be inherited from the time change function $t(x \mid \theta)$, which here would be linear: $t(x \mid \theta)=x / p$ and $\theta=p$, a scalar. We shall take $a(0 \mid \omega)=1$ if $x=0$ is the earliest time point on our scale, so that amplitude is inherited from $g_{0}$.

Similar results, and in particular identical convergence rates of estimators, are obtained for a variety of processes $X_{i}$ that are weakly stationary and weakly independent. They include the cases where the $X_{i}$ s are $n$ points of a homogeneous Poisson process with intensity $\mu^{-1}$ on the positive real line, or $n$ independent random variables, each uniformly distributed on the interval $[0, n \mu]$ or the values of 'jittered grid' data $j \mu+V_{j}$, where the variables $V_{j}$ are independent and identically distributed on a finite interval. In each of these cases the average spacing between adjacent data is asymptotic to $\mu$ as $n \rightarrow \infty$. To avoid aliasing, particularly in cases where the period $\theta$ does not change over time, the $X_{i}$ s should not be located on a grid.

\subsection{Estimators}

To estimate $g_{0}, \omega$ and $\theta$, put

$$
\begin{gathered}
\hat{g}_{0}\{t(x \mid \theta) \mid \theta, \omega\}=\sum_{i} a\left(X_{i} \mid \omega\right)^{-1} Y_{i} K_{i}(x \mid \theta) / \sum_{i} K_{i}(x \mid \theta), \\
S(\theta, \omega)=\sum_{i}\left[Y_{i}-a\left(X_{i} \mid \omega\right) \hat{g}_{0}\left\{t\left(X_{i} \mid \theta\right) \mid \theta, \omega\right\}\right]^{2},
\end{gathered}
$$

where $K_{i}(x \mid \theta)=K\left[\left\{x(\theta)-X_{i}(\theta)\right\} / h\right], K$ is a kernel function, $h$ is a bandwidth,

$$
\begin{gathered}
x(\theta)=t(x \mid \theta)-\lfloor t(x \mid \theta)\rfloor, \\
X_{i}(\theta)=t\left(X_{i} \mid \theta\right)-\left\lfloor t\left(X_{i} \mid \theta\right)\right\rfloor,
\end{gathered}
$$

and $\lfloor u\rfloor$ denotes the largest integer strictly less than $u$.

Let $(\theta, \omega)=(\hat{\theta}, \hat{\omega})$ be the minimizer of $S(\theta, \omega)$. Then, potentially using, to construct $\hat{g}_{0}$, a bandwidth which is different from that employed at equations (9) and (10), our estimator of $g_{0}$ is $\hat{g}_{0}(\cdot \mid \hat{\theta}, \hat{\omega})$. Estimators of the time change function $t_{x}=t\left(x \mid \theta^{0}\right)$ and amplitude function $a_{x}=a\left(x \mid \omega^{0}\right)$ are given by $\hat{t}_{x}=t(x \mid \hat{\theta})$ and $\hat{a}_{x}=a(x \mid \hat{\omega})$ respectively. We estimate $g$, defined at equation (2), as $\hat{g}(x)=\hat{a}_{x} \hat{g}_{0}\left(\hat{t}_{x}\right)$.

\subsection{Hypothesis tests}

We illustrate our approach by considering a particular case, where

(a) $a_{x} \equiv 1$, so that the amplitude does not alter, 
(b) $t_{x}$ is given by equation (4) or, equivalently, the period evolves linearly, as $1 / t_{x}^{\prime}=\theta_{1}+\theta_{2} x$, and

(c) we test $H_{0}: \theta_{2}=0$ against $H_{1}: \theta_{2} \neq 0$ or $H_{2}: \theta_{2}>0$, i.e. we test the null hypothesis of perfect periodicity against the alternative of linearly changing period or linearly increasing period respectively.

Modification of cases (a)-(c) for other contexts is straightforward.

The suggested procedure is as follows.

Step 1: estimate $\theta=\left(\theta_{1}, \theta_{2}\right)$ and $g_{0}$, and thence $g$, under $H_{1}$.

Step 2: compute residuals by subtracting the estimator of $g\left(X_{i}\right)$ from $Y_{i}$.

Step 3: standardize the residuals by correcting for scale and centring. Scale correction would be particularly appropriate if standard deviation estimates were available for each observation; this is sometimes the case for star brightness data (see for example Hall et al. (2000)).

Step 4: using steps 1-3, and perhaps employing a parametric estimate for $g_{0}$ that is suggested by the non-parametric estimate, simulate under $H_{0}$.

Step 5: using the technique of step 4, and the percentile method bootstrap, calculate an upper critical point for the distribution of $\left|\hat{\theta}_{2}\right|$, if we are testing against $H_{1}$, or for the distribution of $\hat{\theta}_{2}$, if we are testing against $\mathrm{H}_{2}$.

Step 6: reject $H_{1}$ or $H_{2}$ if the observed value of $\left|\hat{\theta}_{2}\right|$ or $\hat{\theta}_{2}$ respectively exceeds the upper critical point.

When implementing the bootstrap in step 5 we condition on the observation times $X_{i}$, and so the resampling operation involves resampling only from the collection of standardized residuals. If we have standard deviation estimates available, then, having drawn a residual that we want to attach to the function value at time $X_{i}$, we first rescale it by multiplying by the standard deviation estimate that is associated with that time.

\section{Numerical properties}

\subsection{Summarizing remark}

We shall illustrate the performance and properties of our inferential method for evolving periodic functions in four settings: a model with evolving period and amplitude, where data are generated by using simulation; a model with evolving period, where data are generated by using a combination of simulated and real data; a real data set, where earlier analysis was under the assumption that the period was constant; the data for the Mira variable R Hydrae, which were described in Section 1. Throughout we use a Nadaraya-Watson non-parametric regression estimator (9) with Epanechnikov kernel. We constrain the estimator to be periodic on the interval $[0,1]$ using data on $[-h, 1+h]$ by periodic extension, where $h$ is a bandwidth. The choice of bandwidth has virtually negligible effect, and so we set $h=0.1$ throughout.

\subsection{Simulation study with synthetic observation times}

Here the model is that at equation (2), with the linear model (4) for period, the linear model (7) for amplitude and $g_{0}$ given by $g_{0}(x)=\sin (2 \pi x)$ on the interval [0,1], extended to the positive real line by periodicity. This simple choice of $g_{0}$ is typical of light curve functions for certain variable stars having non-constant periods and amplitudes. See, for example, the case of R Hydrae in Section 3.5. We took the design points $X_{i}$ to be uniformly distributed on the interval $[0, n]$, with sample size $n=250,500,1000,1500$. The parameters for the amplitude and period were chosen to be $\omega_{1}=1 / n, \theta_{1}=2$ and $\theta_{2}=2 / n$, in accordance with the theoretical setting that was described in Sections 2.2 and 2.3. Simulations with larger values of $\theta_{1}$, e.g. $\theta_{1}=50$ or $\theta_{1}=100$, lead to 
Table 1. Biases and variances for the model with linearly evolving period and amplitude treated in Section 3.2

\begin{tabular}{|c|c|c|c|c|c|c|}
\hline$n$ & $b\left(\hat{\omega}_{1}\right)$ & $b\left(\hat{\theta}_{1}\right)$ & $b\left(\hat{\theta}_{2}\right)$ & $n^{3} \operatorname{var}\left(\hat{\omega}_{1}\right)$ & $n^{3} \operatorname{var}\left(\hat{\theta}_{1}\right)$ & $n^{5} \operatorname{var}\left(\hat{\theta}_{2}\right)$ \\
\hline \multicolumn{7}{|c|}{$\sigma=0.05$} \\
\hline 250 & $4.92 \times 10^{-4}$ & $1.73 \times 10^{-5}$ & $-1.37 \times 10^{-7}$ & 0.38 & 1.61 & 7.68 \\
\hline 500 & $2.52 \times 10^{-4}$ & $1.72 \times 10^{-6}$ & $-1.19 \times 10^{-8}$ & 0.38 & 1.31 & 5.63 \\
\hline 1000 & $1.26 \times 10^{-4}$ & $-7.00 \times 10^{-7}$ & $1.86 \times 10^{-9}$ & 0.39 & 0.97 & 3.91 \\
\hline 1500 & $8.34 \times 10^{-5}$ & $-5.33 \times 10^{-7}$ & $1.91 \times 10^{-10}$ & 0.35 & 1.10 & 4.49 \\
\hline \multicolumn{7}{|c|}{$\sigma=0.20$} \\
\hline 250 & $4.84 \times 10^{-4}$ & $1.09 \times 10^{-5}$ & $-5.29 \times 10^{-8}$ & 3.28 & 14.11 & 60.35 \\
\hline 500 & $2.29 \times 10^{-4}$ & $7.54 \times 10^{-6}$ & $-2.83 \times 10^{-8}$ & 3.04 & 12.90 & 57.55 \\
\hline 1000 & $1.27 \times 10^{-4}$ & $4.60 \times 10^{-6}$ & $-8.37 \times 10^{-9}$ & 3.38 & 12.65 & 55.11 \\
\hline 1500 & $8.33 \times 10^{-5}$ & $-1.27 \times 10^{-7}$ & $-1.79 \times 10^{-9}$ & 3.05 & 12.36 & 56.27 \\
\hline
\end{tabular}

similar results. However, small values of $\theta_{1}$ are believed to be more difficult to handle, since fewer observations per period are then available. The errors $\varepsilon_{i}$ in model (8) were taken to be normal $N\left(0, \sigma^{2}\right)$, with $\sigma=0.05$ and $\sigma=0.20$. Biases and variances are calculated by averaging over 500 simulations.

In Table 1 we report the biases of the various estimators, e.g. $b\left(\hat{\omega}_{1}\right)=\overline{\hat{\omega}}_{1}-\omega_{1}$. The biases and variances decrease with increasing sample size. The values of $n^{3 / 2}\left|b\left(\hat{\omega}_{1}\right)\right|, n^{j+1 / 2}\left|b\left(\hat{\theta}_{j}\right)\right|$, $n^{3} \operatorname{var}\left(\hat{\omega}_{1}\right)$ and $n^{2 j+1} \operatorname{var}\left(\hat{\theta}_{j}\right)$ are bounded as $n$ increases.

\subsection{Simulation study with observation times taken from real data}

Here we borrowed the irregular design points $X_{i}$ from the star HIP 023743, which is discussed in Section 3.4, with sample size $n=129$ and data distributed on the interval $[0,1283]$ days. In other respects the data were generated as indicated at equation (2), with the linear model (4) for the period and a constant unit amplitude. Our goal is to illustrate properties of the hypothesis test that was described in Section 2.6. Again, we took $g_{0}$ to be given by $g_{0}(x)=\sin (2 \pi x)$ on the interval $[0,1]$, extended to the positive real line by periodicity. The parameters for the linearly evolving period are chosen to be $\theta_{1}=2$ and $\theta_{2}=0,10^{-10}, 10^{-9}, \ldots, 10^{-5}$. The errors $\varepsilon_{i}$ in model (8) are taken to be normal $N\left(0, \sigma^{2}\right)$, with $\sigma=0.05$ or $\sigma=0.20$. The level for the percentile method bootstrap test is set to 5\%. We create 400 bootstrap samples as described in Section 2.6 and use Fourier series methods to estimate $g_{0}$. Empirical levels and powers are calculated over 500 simulations and are reported in Table 2. We see that the empirical level of our test of $H_{0}: \theta_{2}=0$ against $H_{1}: \theta_{2} \neq 0$ is close to the $5 \%$ nominal level. The empirical power reveals that departures from the null hypothesis of the order of $10^{-5}$ can be detected in this setting.

Table 2. Empirical values of the significance level and power for the model with linearly evolving period treated in part of Section 3.3

\begin{tabular}{|lccccccc|}
\hline$\sigma$ & \multicolumn{7}{c|}{ Rejection rates (\%) for the following values of $\theta_{2}:$} \\
\cline { 2 - 7 } & $\theta_{2}=0$ & $\theta_{2}=10^{-10}$ & $\theta_{2}=10^{-9}$ & $\theta_{2}=10^{-8}$ & $\theta_{2}=10^{-7}$ & $\theta_{2}=10^{-6}$ & $\theta_{2}=10^{-5}$ \\
\hline & & & & & & \\
0.05 & 4.8 & 5.2 & 5.0 & 6.6 & 21.4 & 100.0 & 100.0 \\
0.20 & 5.4 & 5.4 & 6.0 & 7.0 & 9.4 & 42.8 & 100.0 \\
\hline
\end{tabular}




\subsection{First real data example}

Here we consider brightness data $Y_{i}(i=1, \ldots, 129)$ on the star HIP 023743 from the Hipparcos photometry. This star, of the RS CVn type, has been studied before under the assumption that the period is constant, estimated to be 80.9 days. See Eyer and Genton (1999), who used a robust wave variogram approach for inference. On the basis of model (2) with constant period and amplitude, we obtain an estimate of $\hat{\theta}_{1}=81.36$ days for the period. The small difference between those two estimates can be partly attributed to the fact that in the present paper we do not use robust procedures.

If we allow for a linearly evolving period in model (2), we obtain the estimates $\hat{\theta}_{1}=79.44$ and $\hat{\theta}_{2}=3.146 \times 10^{-3}$. Using the hypothesis testing method that was outlined in Section 2.6, this value of $\theta_{2}$ is seen to be highly significantly different from 0 , at the $5 \%$ level. The period of HIP 023743 was apparently increasing, at a rate of about 1.15 days per year, during the observation time interval.

\subsection{Second real data example}

We now return to the brightness data $Y_{i}(i=1, \ldots, 2315)$ on the Mira variable R Hydrae, which were depicted in Fig. 1. (The observation times of these data show significant randomness, although, since each is confined to only a small portion of night hours, the times may appear, from a distance, to be located at points of a grid.) First, we fit model (2) with linearly evolving period and amplitude to the $1900-1950$ data set, and we obtain the estimates $\hat{\omega}_{1}=3.694 \times 10^{-7}$, $\hat{\theta}_{1}=419.3$ and $\hat{\theta}_{2}=-1.441 \times 10^{-3}$. This corresponds effectively to a decrease in the period from about 419 to 394 days during 1900-1950, but accompanied by an increase in the semiamplitude from about 1.707 to 1.718 magnitude. Fig. 2(a) depicts the result of folding the R Hydrae data by using the model and estimates that were noted above. We have superimposed a non-parametric estimate of $g_{0}$ (the full curve). The corresponding minimum of the criterion $S$, which is defined by equation (10), is 925.2 .

If, instead, we fold the data with the parameters corresponding to the changes in period and amplitude that were reported by Zijlstra et al. (2002), we obtain a result which does not reflect the data set quite as well; see Fig. 2(b). The corresponding minimum of $S$ is 2908.

Next, we investigate potential departures from a constant period of 385 days during 1950 2001. We fit model (2) with linearly evolving period and constant amplitude, and we obtain the estimates $\hat{\theta}_{1}=394.1$ and $\hat{\theta}_{2}=-5.872 \times 10^{-3}$. Using the hypothesis testing method that was outlined in Section 2.6, this value of $\theta_{2}$ is seen to be highly significantly different from 0 , at the $5 \%$ level. Therefore, one could argue that the period of $\mathrm{R}$ Hydrae further decreased during 1950-2001 to about 383 days in 2001. Fig. 2(c) depicts the result of folding the R Hydrae data by using the model and estimates that were noted above.

\section{Theoretical properties}

For transparency and brevity we shall treat polynomial models for time change and amplitude, and also a simple model for the distribution of the $X_{i}$ s. See assumptions (a)-(f) below. Modified versions of our argument are valid for any of the models for $t_{x}$ and $a_{x}$ that were introduced in Sections 2.2 and 2.3; the arguments give estimators with the same convergence rates as those discussed below. Likewise, the convergence rates that we give continue to hold if the model for the $X_{i}$ s is altered to either of models (a) and (c) that were discussed in Section 2.4.

However, for the $t_{x}$ - and $a_{x}$-models that we consider in assumptions (b) and (c) below, and for the alternative models that were discussed in Sections 2.2 and 2.3, our convergence rates are contingent on the assumption that $\sup _{j}\left(n^{j-1}\left|\theta_{j}\right|\right)$ and $\sup _{j}\left(n^{j}\left|\omega_{j}\right|\right)$ are bounded 
in $n$. This constrains us away from the unrealistic setting where the period and amplitude alter by an order of magnitude as the length of the observation interval $[0, n]$ increases. If the constraint should fail then convergence rates can be quite different from those given below.

In assumptions (b) and (c) below we actually impose a negligibility condition, specifically that $\sup _{j}\left(n^{j-1}\left|\theta_{j}\right|\right)$ and $\sup _{j}\left(n^{j}\left|\omega_{j}\right|\right)$ both converge to 0 as $n \rightarrow \infty$. This ensures that the covariance matrix of the joint estimators of $\theta$ and $\omega$ has a simple asymptotic form. Rates do not alter if $\sup _{j}\left(n^{j-1}\left|\theta_{j}\right|\right)$ and $\sup _{j}\left(n^{j}\left|\omega_{j}\right|\right)$ are merely bounded, but the limiting covariance matrix is different.

Next we state our assumptions. Suppose that the data pairs $\left(X_{i}, Y_{i}\right)$ are generated by model (8), and that the observation times $X_{i}$ all lie in $[0, n]$. We ask the following.

(a) For $g_{0}: g_{0}$ is periodic with minimal period equal to 1 , has two bounded derivatives and is not identically constant.

(b) For $t_{x}: t_{x}=t(x \mid \theta)$ is given by the $k$-parameter polynomial model (6), where $\theta_{1}>0$ is fixed and $\theta_{2}, \ldots, \theta_{k}$ vary with $n$, satisfying $\sup _{2 \leqslant j \leqslant k}\left(n^{j-1}\left|\theta_{j}\right|\right) \rightarrow 0$.

(c) For $a_{x}: a_{x}=a(x \mid \omega)$ is given by the $l$-parameter polynomial model (7), where $\omega_{1}, \ldots, \omega_{l}$ vary with $n$ and satisfy $\sup _{1 \leqslant j \leqslant l}\left(n^{j}\left|\omega_{j}\right|\right) \rightarrow 0$.

(d) For the error distribution: the errors $\varepsilon_{i}$ are independent and identically distributed with zero mean, $E\left(|\varepsilon|^{c}\right)<\infty$ for some $c>2$, and $\operatorname{var}\left(\varepsilon_{i}\right)=\sigma^{2}$.

(e) For the observation times: $X_{1}, \ldots, X_{n}$ are generated by model (b) from Section 2.4, and in particular represent values of $n$ independent and identically distributed random variables that are uniformly distributed on $[0, n]$.

(f) For $h$ and $K: h=c n^{-d}$, where $c>0$ and $\frac{1}{4}<d<\frac{1}{3}$, and $K$ is a symmetric, compactly supported density with three continuous derivatives on the real line.

Let $U$ denote a random variable having the uniform distribution on [0,1], define $J_{1}=-\left(\theta_{1}^{0}\right)^{-2}$ and $J_{r}=1$ if $r \geqslant 2$, and let $\Sigma$ denote the symmetric $(k+l) \times(k+l)$ matrix of which the $(r, s)$ th component is

$$
J_{r} J_{S} E\left\{g_{0}^{\prime}(U)^{2}\right\}\left\{(r+s+1)^{-1}-(r+1)^{-1}(s+1)^{-1}\right\}
$$

if $1 \leqslant r, s \leqslant k$,

$$
E\left\{g_{0}(U)^{2}\right\}\left\{\left(r_{1}+s_{1}+1\right)^{-1}-\left(r_{1}+1\right)^{-1}\left(s_{1}+1\right)^{-1}\right\}
$$

if $(r, s)=\left(k+r_{1}, k+s_{1}\right)$ with $1 \leqslant r_{1}, s_{1} \leqslant l$ and

$$
-J_{r} E\left\{g_{0}(U) g_{0}^{\prime}(U)\right\}\left\{\left(r+s_{1}+1\right)^{-1}-(r+1)^{-1}\left(s_{1}+1\right)^{-1}\right\}
$$

if $1 \leqslant r \leqslant k$ and $s=k+s_{1}$ with $1 \leqslant s_{1} \leqslant l$. We further assume that $\Sigma$ is non-singular, which imposes minor conditions on the possible values that can be taken by the moments $E\left\{g_{0}(U)^{i} g_{0}^{\prime}(U)^{j}\right\}$ for $(i, j)=(0,2),(2,0),(1,1)$. Define

$$
\begin{aligned}
& \hat{\Theta}=\left(n\left(\hat{\theta}_{1}-\theta_{1}^{0}\right), \ldots, n^{k}\left(\hat{\theta}_{k}-\theta_{k}^{0}\right)\right)^{\mathrm{T}}, \\
& \hat{\Omega}=\left(n\left(\hat{\omega}_{1}-\omega_{1}^{0}\right), \ldots, n^{l}\left(\hat{\omega}_{l}-\omega_{l}^{0}\right)\right)^{\mathrm{T}},
\end{aligned}
$$

and put $\hat{\Phi}=\left(\hat{\Theta}^{\mathrm{T}}, \hat{\Omega}^{\mathrm{T}}\right)^{\mathrm{T}}$, a column vector of length $k+l$.

Theorem 1. Assuming requirements (a)-(f) above, and that $\Sigma$ is non-singular, $n^{1 / 2} \hat{\Phi}$ is asymptotically normally distributed with zero mean and variance matrix $\Sigma^{-1} \sigma^{2}$. 


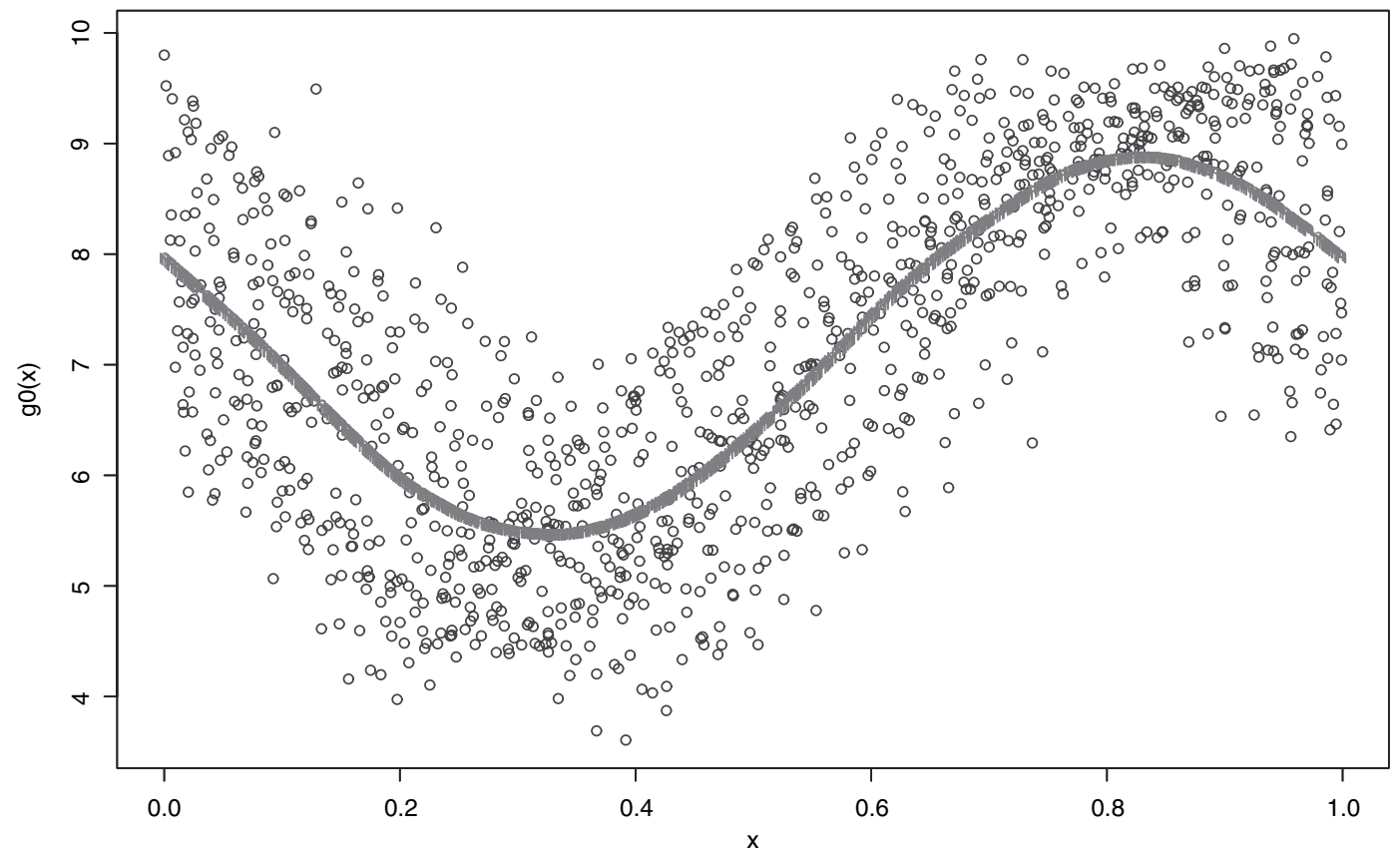

(a)

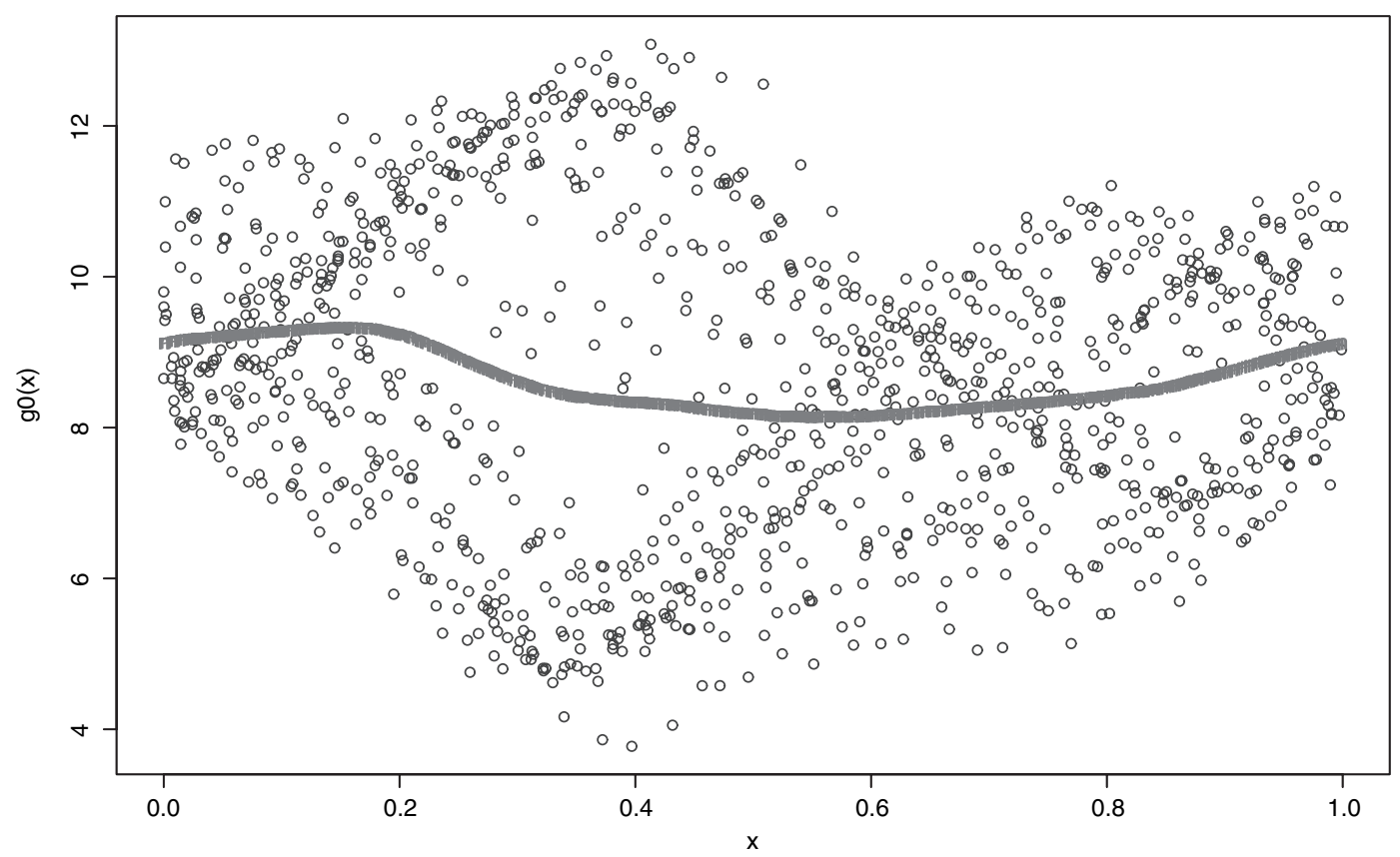

(b)

Fig. 2. Folded plot with non-parametric estimation of $g_{0}$ for the Mira variable R Hydrae: (a) after fitting linearly increasing period and amplitude to the 1900-1950 data set by using the method suggested in this paper; (b) after fitting linearly increasing period and amplitude to the 1900-1950 data set by using an alternative method; (c) after fitting linearly increasing period to the 1950-2001 data set by using the method suggested in this paper 


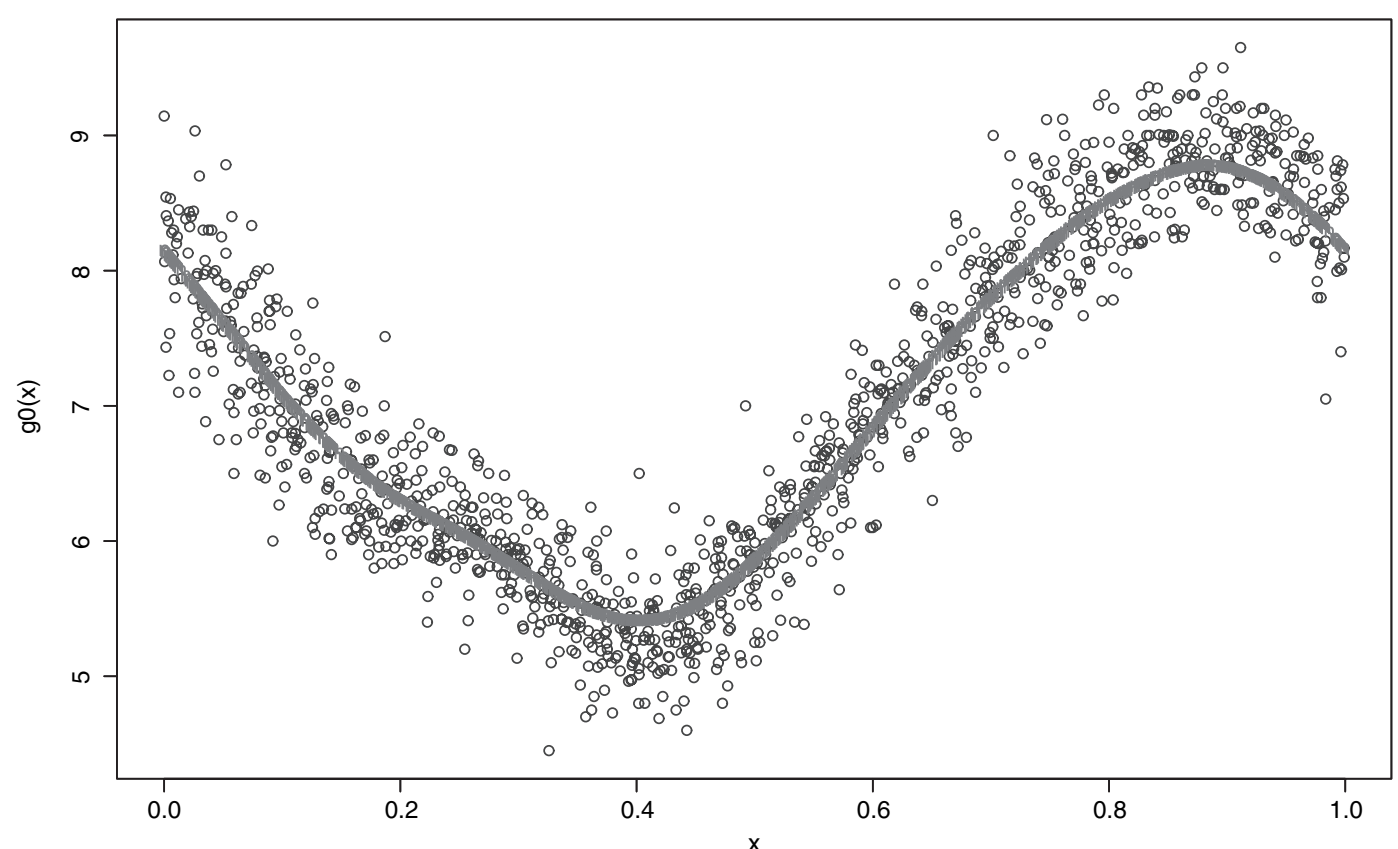

(c)

Fig. 2 (continued)

Since $\hat{\Phi}=\left(\hat{\Theta}^{\mathrm{T}}, \hat{\Omega}^{\mathrm{T}}\right)^{\mathrm{T}}$ then result (14) implies that

$$
\begin{array}{ll}
n^{j}\left(\hat{\theta}_{j}-\theta_{j}^{0}\right)=O_{p}\left(n^{-1 / 2}\right) & \text { for } 1 \leqslant j \leqslant k, \\
n^{j}\left(\hat{\omega}_{j}-\omega_{j}^{0}\right)=O_{p}\left(n^{-1 / 2}\right) & \text { for } 1 \leqslant j \leqslant l .
\end{array}
$$

These results continue to hold if we drop the condition of identical distribution in assumption (d), assuming instead, for example, that the errors $\varepsilon_{i}$ are distributed as $\sigma_{i} \varepsilon$, where $\sup _{i}\left(\sigma_{i}\right)<\infty$ and $E\left(|\varepsilon|^{c}\right)<\infty$ for some $c>2$. Likewise, conditions (15) and (16) continue to hold if the conditions $\sup _{2 \leqslant j \leqslant k}\left(n^{j-1}\left|\theta_{j}\right|\right) \rightarrow 0$ and $\sup _{1 \leqslant j \leqslant l}\left(n^{j}\left|\omega_{j}\right|\right) \rightarrow 0$, in assumptions (b) and (c) respectively, are replaced by the assumptions that $\sup _{2 \leqslant j \leqslant k}\left(n^{j-1}\left|\theta_{j}\right|\right)$ and $\sup _{1 \leqslant j \leqslant l}\left(n^{j}\left|\omega_{j}\right|\right)$ are bounded as $n \rightarrow \infty$.

These convergence rates are so fast that first-order asymptotic theory for our estimator of $g_{0}$ is identical to its counterpart that would obtain if the values of $\theta^{0}$ and $\omega^{0}$ were known. Indeed, if $h$ is chosen so that condition (15)-(16) holds, for example, satisfying assumption (f), and if we then select a new bandwidth $h=h_{1}$, of the size that would be appropriate for estimating $g_{0}$ nonparametrically, then $\hat{g}_{0}(\cdot \mid \hat{\theta}, \hat{\omega})$, which is defined in Section 2.5, satisfies $\hat{g}_{0}(\cdot \mid \hat{\theta}, \hat{\omega})=\hat{g}_{0}\left(\cdot \mid \theta^{0}, \omega^{0}\right)+$ $o_{p}\left\{\left(n h_{1}\right)^{-1 / 2}\right\}$. An appropriate choice of $h_{1}$ is one that satisfies $h_{1} \geqslant n^{-1 / 2+\delta}$ for some $\delta>0$.

Standard methods for deriving bootstrap properties may be used to show that under mild additional assumptions, in particular continuity of the error distribution, the hypothesis tests that were discussed in Section 2.6 have asymptotically correct coverage. Furthermore, those tests can distinguish alternatives that are distant $n^{-3 / 2}$ from the null hypothesis.

To formalize the latter result, let us take the specific example that was given in Section 2.6. In particular, assume that $a_{x} \equiv 1$ and $t_{x}$ is given by equation (4) with $\theta_{1}>0$ fixed and $\theta_{2}=c n^{-3 / 2}$, for $c$ fixed. Take $c \neq 0$ in the case of a test against $H_{1}$, and $c>0$ for a test against $H_{2}$. Let $P_{c}$ denote probability measure under $H_{j}$, for $j=1$ or $j=2$ and for a particular value of $c$. Then, 


$$
\left.\lim _{|c| \rightarrow \infty} \liminf _{n \rightarrow \infty}\left\{P_{c} \text { (test rejects } H_{0} \mid H_{j} \text { is true }\right)\right\}=1 .
$$

This result is intuitively clear from result (14).

\section{Acknowledgements}

We are grateful to three reviewers for helpful comments. This work was started while the first author was visiting the Centre for Mathematics and Its Applications, Australian National University, whose kind hospitality and financial support is gratefully acknowledged. The work of Genton was also partially supported by National Science Foundation grant DMS-0504896.

\section{Appendix A: Derivation of asymptotic properties}

\section{A.1. Preliminaries for proof}

In view of model (8),

$$
Y_{j}-a\left(X_{j} \mid \omega\right) \hat{g}_{0}\left\{t\left(X_{j} \mid \theta\right) \mid \theta, \omega\right\}=R_{j}(\theta, \omega)+S_{j}(\theta, \omega)
$$

where

$$
\begin{gathered}
R_{j}(\theta, \omega)=R\left(X_{j} \mid \theta, \omega\right), \\
S_{j}(\theta, \omega)=\varepsilon_{j}-S\left(X_{j} \mid \theta, \omega\right), \\
R(x \mid \theta, \omega)=a\left(x \mid \omega^{0}\right) g_{0}\left\{t\left(x \mid \theta^{0}\right)\right\}-a(x \mid \omega) \frac{B(x \mid \theta, \omega)}{A(x \mid \theta)}, \\
S(x \mid \theta, \omega)=a(x \mid \omega) \frac{D(x \mid \theta, \omega)}{A(x \mid \theta)}, \\
A(x \mid \theta)=\sum_{i=1}^{\nu} K_{i}(x \mid \theta), \\
B(x \mid \theta, \omega)=\sum_{i=1}^{\nu} \frac{a\left(X_{i} \mid \omega^{0}\right) g_{0}\left\{t\left(X_{i} \mid \theta^{0}\right)\right\}}{a\left(X_{i} \mid \omega\right)} K_{i}(x \mid \theta), \\
D(x \mid \theta, \omega)=\sum_{i=1}^{\nu} \frac{\varepsilon_{i}}{a\left(X_{i} \mid \omega\right)} K_{i}(x \mid \theta) .
\end{gathered}
$$

Arguing as in Hall et al. (2000) it may be shown that the contribution that is made by $S\left(X_{j} \mid \theta, \omega\right)$ to $S_{j}(\theta, \omega)$ is asymptotically negligible, as also is the stochastic contribution that is made by $R(x \mid \theta, \omega)$, i.e., defining $\pi_{A}(x \mid \theta, \omega)=E\{A(x \mid \theta, \omega)\}$ and $\pi_{B}(x \mid \theta, \omega)=E\{B(x \mid \theta, \omega)\}$, putting

$$
\pi_{R}(x \mid \theta, \omega)=a\left(x \mid \omega^{0}\right) g_{0}\left\{t\left(x \mid \theta^{0}\right)\right\}-a(x \mid \omega) \frac{\pi_{B}(x \mid \theta, \omega)}{\pi_{A}(x \mid \theta)},
$$

and taking $(\hat{\theta}, \hat{\omega})$ to be the minimizer of $\Sigma_{j}\left\{\varepsilon_{j}-\pi_{R}\left(X_{j} \mid \theta, \omega\right)\right\}^{2}$ rather than of $S(\theta, \omega)$ at equation (10), $\left(\hat{\theta}-\theta^{0}, \hat{\omega}-\omega^{0}\right)$ has the sought asymptotic normal distribution.

Moreover, also as in Hall et al. $(2000), \pi_{R}(x \mid \theta, \omega)=p(x)^{\mathrm{T}} \Theta+q(x)^{\mathrm{T}} \Omega+\ldots$, where $p(x)$ and $q(x)$ denote a $k$-vector and an $l$-vector respectively, and, from this point down to equation (23) below, '...' indicates terms that are quadratic in $\Theta$ and/or $\Omega$. Up to terms that do not influence the limiting distributions of $\hat{\Theta}$ and $\hat{\Omega}$, those quantities minimize

$$
\sum_{j=1}^{\nu}\left\{\varepsilon_{j}-p\left(X_{j}\right)^{\mathrm{T}} \Theta-q\left(X_{j}\right)^{\mathrm{T}} \Omega\right\}^{2} .
$$

Thus, $n^{1 / 2}\left(\hat{\Theta}^{\mathrm{T}}, \hat{\Omega}^{\mathrm{T}}\right)^{\mathrm{T}}$ is asymptotically normally distributed with zero means and variance $\Sigma^{-1} \sigma^{2}$, where

$$
\Sigma=E\left\{\left(p(X)^{\mathrm{T}}, q(X)^{\mathrm{T}}\right)^{\mathrm{T}}\left(p(X)^{\mathrm{T}}, q(X)^{\mathrm{T}}\right)\right\} .
$$

We shall show in the next section that $p=\left(p_{1}, \ldots, p_{k}\right)^{\mathrm{T}}$ and $q=\left(q_{1}, \ldots, q_{l}\right)^{\mathrm{T}}$, where 


$$
\begin{gathered}
p_{r}(x)=-J_{r}\left\{(x / n)^{r}-(r+1)^{-1}\right\} g_{0}^{\prime}\left\{t\left(x \mid \theta^{0}\right)\right\}, \\
q_{s}(x)=\left\{(x / n)^{s}-(s+1)^{-1}\right\} g_{0}\left\{t\left(x \mid \theta^{0}\right)\right\},
\end{gathered}
$$

for $1 \leqslant r \leqslant k$ and $1 \leqslant s \leqslant l$. Substituting these definitions into equation (17) we obtain, up to a term which converges to 0 as $n \rightarrow \infty$, the matrix $\Sigma$ that is defined at expressions (11)-(13).

\section{A.2. Derivation of $p$ and $q$ at equations (18) and (19)}

Without loss of generality, $K$ is supported on $[-1,1]$. Given $\eta \in\left(0, \frac{1}{2}\right)$ and $c>1$, let $\mathcal{I}_{n}(\eta)$ denote the set of $x \in[0, c n]$ such that $t\left(x \mid \theta^{0}\right)$ is at least $\eta$ from the nearest integer. Put $\dot{t}(x \mid \theta)=\partial t(x \mid \theta) / \partial \theta$, and let $C$ denote a generic positive constant, not depending on $n$ but potentially different at each appearance. Note that $\left|t(x \mid \theta)-t\left(x \mid \theta^{0}\right)\right| \leqslant C\left\|\theta-\theta^{0}\right\|$. Therefore, if

(a) $\left\|\theta-\theta^{0}\right\| \leqslant C \eta$ and

(b) $x \in \mathcal{I}_{n}(\eta)$

then $\lfloor t(x \mid \theta)\rfloor=\left\lfloor t\left(x \mid \theta^{0}\right)\right\rfloor$, and so

$$
t(x \mid \theta)-\lfloor t(x \mid \theta)\rfloor-\left\{t\left(x \mid \theta^{0}\right)-\left\lfloor t\left(x \mid \theta^{0}\right)\right\rfloor\right\}=t(x \mid \theta)-t\left(x \mid \theta^{0}\right) .
$$

Provided that

$$
K\left[\frac{t\left(x_{1} \mid \theta\right)-\left\lfloor t\left(x_{1} \mid \theta\right)\right\rfloor-\left\{t\left(x_{2} \mid \theta\right)-\left\lfloor t\left(x_{2} \mid \theta\right)\right\rfloor\right\}}{h}\right] \neq 0,
$$

we have $-h<t\left(x_{1} \mid \theta\right)-\left\lfloor t\left(x_{1} \mid \theta\right)\right\rfloor-\left\{t\left(x_{2} \mid \theta\right)-\left\lfloor t\left(x_{2} \mid \theta\right)\right\rfloor\right\}<h$, and if also $t\left(x_{1} \mid \theta\right)-\left\lfloor t\left(x_{1} \mid \theta\right)\right\rfloor \in[C h, 1-C h]$ then $x_{2} \in \mathcal{I}_{n}(h)$. Therefore, if

(c) $x \in[C h, 1-C h]$ and

(d) $K_{i}\left(x \mid \theta_{1}\right) \neq 0$, where $\theta_{1}$ denotes either $\theta$ or $\theta^{0}$,

then $X_{i} \in \mathcal{I}_{n}(h)$. Hence, using the fact that (a) and (b) together imply equation (20), we deduce that if (c), (d) and

(e) $\left\|\theta-\theta^{0}\right\| \leqslant C h$ hold,

then

$$
\begin{aligned}
K_{i}(x \mid \theta) & =K\left[\frac{t\left(x \mid \theta^{0}\right)-X_{i}\left(\theta^{0}\right)+t(x \mid \theta)-t\left(x \mid \theta^{0}\right)-\left\{t\left(X_{i} \mid \theta\right)-t\left(X_{i} \mid \theta^{0}\right)\right\}}{h}\right] \\
& =K_{i}\left(x \mid \theta^{0}\right)-h^{-1}\left\{\dot{t}\left(X_{i} \mid \theta^{0}\right)-\dot{t}\left(x \mid \theta^{0}\right)\right\}^{\mathrm{T}}\left(\theta-\theta^{0}\right) K_{i}^{\prime}\left(x \mid \theta^{0}\right)+\ldots,
\end{aligned}
$$

where $K_{i}^{\prime}(x \mid \theta)$ is defined to equal $K^{\prime}\left[\left\{x(\theta)-X_{i}(\theta)\right\} / h\right]$.

By Taylor series expansion of $a\left(X_{i} \mid \omega\right)^{-1}$ we may show that

$$
R(x \mid \theta, \omega)=a\left(x \mid \omega^{0}\right)\left[g_{0}\left\{t\left(x \mid \theta^{0}\right)\right\}-\frac{G(x \mid \theta)}{A(x \mid \theta)}\right]+Q\left(x \mid \theta^{0}, \omega^{0}\right)\left(\omega-\omega^{0}\right)+\ldots,
$$

where

$$
\begin{gathered}
Q(x \mid \theta, \omega)=\frac{G(x \mid \theta)}{A(x \mid \theta)} \dot{a}(x \mid \omega)-\frac{H(x \mid \theta)}{A(x \mid \theta)}, \\
G(x \mid \theta)=\sum_{i=1}^{\nu} g_{0}\left\{t\left(X_{i} \mid \theta^{0}\right)\right\} K_{i}(x \mid \theta), \\
H(x \mid \theta)=\sum_{i=1}^{\nu} \frac{g_{0}\left\{t\left(X_{i} \mid \theta^{0}\right)\right\} \dot{a}\left(X_{i} \mid \omega^{0}\right)}{a\left(X_{i} \mid \omega^{0}\right)} K_{i}(x \mid \theta) .
\end{gathered}
$$

Using equation (21) to expand $G(x \mid \theta) / A(x \mid \theta)$ by Taylor series, in equation (22), about $\theta=\theta^{0}$, and defining 


$$
\begin{gathered}
L(x \mid \theta)=\sum_{i=1}^{\nu}\left\{\dot{t}\left(X_{i} \mid \theta\right)-\dot{t}(x \mid \theta)\right\} g_{0}\left\{t\left(X_{i} \mid \theta\right)\right\} K_{i}^{\prime}(x \mid \theta), \\
N(x \mid \theta)=\sum_{i=1}^{\nu}\left\{\dot{t}\left(X_{i} \mid \theta\right)-\dot{t}(x \mid \theta)\right\} K_{i}^{\prime}(x \mid \theta), \\
P(x \mid \theta, \omega)=a(x \mid \theta, \omega)\left\{\frac{L(x \mid \theta)}{A(x \mid \theta)}-\frac{G(x \mid \theta) N(x \mid \theta)}{A(x \mid \theta)^{2}}\right\}, \\
T(x \mid \theta, \omega)=a(x \mid \omega)\left[g_{0}\{t(x \mid \theta)\}-\frac{G(x \mid \theta)}{A(x \mid \theta)}\right],
\end{gathered}
$$

we may show that

$$
R(x \mid \theta, \omega)=T\left(x \mid \theta^{0}, \omega^{0}\right)+h^{-1} P\left(x \mid \theta^{0}, \omega^{0}\right)^{\mathrm{T}}\left(\theta-\theta^{0}\right)+Q\left(x \mid \theta^{0}, \omega^{0}\right)^{\mathrm{T}}\left(\omega-\omega^{0}\right)+\ldots
$$

Retracing this argument with quantities replaced by their expected values, we obtain

$$
\pi_{R}(x \mid \theta, \omega)=\pi_{T}\left(x \mid \theta^{0}, \omega^{0}\right)+h^{-1} \pi_{P}\left(x \mid \theta^{0}, \omega^{0}\right)^{\mathrm{T}}\left(\theta-\theta^{0}\right)+\pi_{Q}\left(x \mid \theta^{0}, \omega^{0}\right)^{\mathrm{T}}\left(\omega-\omega^{0}\right)+\ldots,
$$

where, for $W$ equal to $G, H, L$ or $N, \pi_{W}=E(W)$, and

$$
\begin{gathered}
\pi_{P}(x \mid \theta, \omega)=a(x \mid \theta, \omega)\left\{\frac{\pi_{L}(x \mid \theta)}{\pi_{A}(x \mid \theta)}-\frac{\pi_{G}(x \mid \theta) \pi_{N}(x \mid \theta)}{\pi_{A}(x \mid \theta)^{2}}\right\}, \\
\pi_{Q}(x \mid \theta, \omega)=\frac{\pi_{G}(x \mid \theta)}{\pi_{A}(x \mid \theta)} \dot{a}(x \mid \omega)-\frac{\pi_{H}(x \mid \theta)}{\pi_{A}(x \mid \theta)}, \\
\pi_{T}(x \mid \theta, \omega)=a(x \mid \omega)\left[g_{0}\{t(x \mid \theta)\}-\frac{\pi_{G}(x \mid \theta)}{\pi_{A}(x \mid \theta)}\right] .
\end{gathered}
$$

Starting from equation (23), conventional bias expansions for kernel estimators show that, with $q_{s}$ defined as at equation (19) and with

$$
\rho(x)=\left(n h^{2}\right)^{-1} a\left(x \mid \theta^{0}, \omega^{0}\right)\left[E\left\{L\left(x \mid \theta^{0}\right)\right\}-g_{0}\left\{t\left(x \mid \theta^{0}\right)\right\} E\left\{N\left(x \mid \theta^{0}\right)\right\}\right],
$$

we have

$$
\pi_{R}(x \mid \theta, \omega)=\rho(x)^{\mathrm{T}}\left(\theta-\theta^{0}\right)+q(x)^{\mathrm{T}} \Omega+\ldots . .
$$

Here the '...' terms consist of quadratic contributions, plus quantities that are of order $h^{2}$ and which, in view of the assumptions that are imposed on the bandwidth, make an asymptotically negligible final contribution.

Write $l_{r}(x)$ for the $r$ th component of $h^{-1} E\left\{L\left(x \mid \theta^{0}\right)\right\}$, multiplied by $-\left(\theta_{1}^{0}\right)^{2}$ in the case $r=1$. Put $t^{\prime}(u \mid \theta)=$ $(\partial / \partial u) t(u \mid \theta)$. If $t\left(x \mid \theta^{0}\right)-\left\lfloor t\left(x \mid \theta^{0}\right)\right\rfloor \in(C h, 1-C h)$ and $c>1$ then $\left\lfloor t\left(x-h u \mid \theta^{0}\right)\right\rfloor=\left\lfloor t\left(x \mid \theta^{0}\right)\right\rfloor$ for all $x \in[0, c n]$ and all $|u| \leqslant 1$, and so

$$
t\left(x-h u \mid \theta^{0}\right)-\left\lfloor t\left(x-h u \mid \theta^{0}\right)\right\rfloor=t\left(x \mid \theta^{0}\right)-\left\lfloor t\left(x \mid \theta^{0}\right)\right\rfloor-h u t^{\prime}\left(x \mid \theta^{0}\right)+o(h|u|) .
$$

This leads to the formula

$$
l_{r}(x)=h \sum_{j=1}^{n-1}\left[g_{0}^{\prime}(v)\left\{t^{-1}\left(j+v \mid \theta^{0}\right)^{r}-x^{r}\right\}+r g_{0}(v) t^{-1}\left(j+v \mid \theta^{0}\right)^{r-1}\right]\left(t^{-1}\right)^{\prime}\left(j+v \mid \theta^{0}\right)+o\left(n^{r+1} h\right),
$$

where $v=v(x)=t\left(x \mid \theta^{0}\right)-\left\lfloor t\left(x \mid \theta^{0}\right)\right\rfloor$. Similarly, writing $n_{r}(x)$ for the $r$ th component of $h^{-1} g_{0}\left\{t\left(x \mid \theta^{0}\right)\right\} \times$ $E\left\{N\left(x \mid \theta^{0}\right)\right\}$, multiplied by $-\left(\theta_{1}^{0}\right)^{2}$ when $r=1$, we obtain

$$
n_{r}(x)=h \sum_{j=1}^{n-1} r g_{0}(v) t^{-1}\left(j+v \mid \theta^{0}\right)^{r-1}\left(t^{-1}\right)^{\prime}\left(j+v \mid \theta^{0}\right)+o\left(n^{r+1} h\right) .
$$

Therefore, defining $\rho_{r}(x)$ to equal the $r$ th component of $\rho(x)$, we deduce that

$$
\rho_{r}(x)=(n h)^{-1} a\left(x \mid \theta^{0}, \omega^{0}\right)\left\{l_{r}(x)-n_{r}(x)\right\}=n^{r} p_{r}(x)+\ldots,
$$

with $p_{r}$ given at equation (18). From this result and equation (24) we see that $\pi_{R}(x \mid \theta, \omega)=p(x)^{\mathrm{T}} \Theta+$ $q(x)^{\mathrm{T}} \Omega+\ldots$, where, from equation (24) the '...' terms make an asympotically negligible final contribution, which establishes equations (18) and (19). 


\section{References}

Aerts, C. and Kolenberg, K. (2005) HD 121190: a cool multiperiodic slowly pulsating B star with moderate rotation. Astronom. Astrophys., 431, 614-622.

Barning, F. J. M. (1963) The numerical analysis of the light-curve of 12 Lacertae. Bull. Astronom. Inst. Nether., $17,22-28$.

de Cat, P. and Aerts, C. (2002) A study of bright southern slowly pulsating B stars, II: The intrinsic frequencies. Astronom. Astrophys., 393, 965-982.

Dahlhaus, R. (1997) Fitting time series models to nonstationary processes. Ann. Statist., 25, 1-37.

DePoy, D. L., Pepper, J., Pogge, R. W., Stutz, A., Pinsonneault, M. and Sellgren, K. (2004) The nature of the variable galactic center source IRS 16SW. Astrophys. J., 617, 1127-1130.

Eyer, L. and Genton, M. G. (1999) Characterization of variable stars by robust wave variograms: an application to Hipparcos mission. Astronom. Astrophys., suppl. ser., 136, 421-428.

Ferraz-Mello, S. (1981) Estimation of periods from unequally spaced observations. Astronom. J., 86, 619-624.

Hall, P. and Li, M. (2006) Using the periodogram to estimate period in nonparametric regression. Biometrika, 93, 411-424.

Hall, P., Reimann, J. and Rice, J. (2000) Nonparametric estimation of a periodic function. Biometrika, 87, 545-557.

Hall, P. and Yin, J. (2003) Nonparametric methods for deconvolving multiperiodic functions. J. R. Statist. Soc. B, 65, 869-886.

Hart, J. D., Koen, C. and Lombard, F. (2007) An analysis of pulsation periods of long period variable stars. Appl. Statist., 56, in the press.

Koen, C. (2005) Statistics of O-C diagrams and period changes. Proc. Astronom. Soc. Pacif. Conf. Ser., 335, 25-36.

Lanza, A. F., Rodonò, M. and Pagano, I. (2004) Multiband modelling of the Sun as a variable star from VIRGO/SoHO data. Astronom. Astrophys., 425, 707-717.

Lomb, N. R. (1976) Least-squares frequency analysis of unequally spaced data. Astrophys. Space Sci., 39, 447-462.

Maffei, P., Ciprini, S. and Tosti, G. (2005) Blue and infrared light curves of the mysterious pre-main-sequence star V582 Mon (KH 15D) from 1955 to 1970. Mnthly Notes R. Astronom. Soc., 357, 1059-1067.

Neumann, M. H. and von Sachs, R. (1997) Wavelet thresholding in anisotropic function classes and application to adaptive estimation of evolutionary spectra. Ann. Statist., 25, 38-76.

Rodler, F. and Guggenberger, E. (2005) Spurious period shifts and changes among variable stars. Proc. Astronom. Soc. Pacif. Conf. Ser., 335, 115-118.

Scargle, J. D. (1982) Studies in astronomical time-series analysis II: statistical aspects of spectral analysis of unevenly spaced data. Astrophys. J., 263, 835-853.

Sterken, C. (2005) The O-C diagram: basic procedures. Proc. Astronom. Soc. Pacif. Conf. Ser., 335, 3-22.

Zijlstra, A. A., Bedding, T. R. and Mattei, J. A. (2002) The evolution of the Mira variable R Hydrae. Mnthly Notes. R. Astronom. Soc., 334, 498-510. 